\title{
Anomalous electron-phonon coupling probed on the surface of superconductor $\mathrm{ZrB}_{12}$
}

\author{
R. Khasanov, ${ }^{1,2,3}$ D. Di Castro, ${ }^{1,4}$ M. Belogolovskii, ${ }^{5}$ Yu. Paderno, ${ }^{6}$ V. Filippov, ${ }^{6}$ R. Brütsch, ${ }^{7}$ and H. Keller ${ }^{1}$ \\ ${ }^{1}$ Physik-Institut der Universität Zürich, Winterthurerstrasse 190, CH-8057, Zürich, Switzerland \\ ${ }^{2}$ DPMC, Université de Genève, 24 Quai Ernest-Ansermet, 1211 Genève 4, Switzerland \\ ${ }^{3}$ Laboratory for Neutron Scattering, ETH Zürich and Paul Scherrer Institut, CH-5232 Villigen PSI, Switzerland \\ ${ }^{4}$ INFM-Coherentia and Dipartimento di Fisica, Università di Roma "La Sapienza," P.le A. Moro 2, I-00185 Roma, Italy \\ ${ }^{5}$ Donetsk Physical and Technical Institute, National Academy of Science of Ukraine, 83114 Donetsk, Ukraine \\ ${ }^{6}$ Institute for Problems of Materials Science, National Academy of Science of Ukraine, 03680 Kiev, Ukraine \\ ${ }^{7}$ Laboratory for Material Behaviour, Paul Scherrer Institut, CH-5232 Villigen PSI, Switzerland
}

(Received 22 September 2005; published 14 December 2005)

\begin{abstract}
Magnetization measurements under hydrostatic pressure up to $10.5 \mathrm{kbar}$ in zirconium dodecaboride $\mathrm{ZrB}_{12}$ superconductor $\left(T_{c} \simeq 6.0 \mathrm{~K}\right.$ at $\left.p=0\right)$ were carried out. A negative pressure effect on $T_{c}$ with $d T_{c} / d p=$ $-0.0225(3) \mathrm{K} / \mathrm{kbar}$ was observed. The electron-phonon coupling constant $\lambda_{\mathrm{el} \text {-ph }}$ decreases with increasing pressure with $d \ln \lambda_{\text {el-ph }} / d p \simeq-0.20 \% / \mathrm{kbar}$. The magnetic field penetration depth $\lambda$ was studied in the Meissner state and, therefore, probes mainly the surface of the sample. The absolute values of $\lambda$ and the superconducting energy gap at ambient pressure and zero temperature were found to be $\lambda(0)=140(30) \mathrm{nm}$ and $\Delta_{0}$ $=1.251(9) \mathrm{meV}$, respectively. $\Delta_{0}$ scales linearly with $T_{c}$ as $2 \Delta_{0} / k_{B} T_{c}=4.79(1)$. The studies of the pressure effect on $\lambda$ reveal that $\lambda^{-2}$ increases with pressure with $d \ln \lambda^{-2}(0) / d p=0.60(23) \% / \mathrm{kbar}$. This effect cannot be explained within the framework of conventional adiabatic electron-phonon pairing, suggesting the possibility that close to the surface an unconventional nonadiabatic character of the electron-phonon coupling takes place.
\end{abstract}

DOI: 10.1103/PhysRevB.72.224509

\section{INTRODUCTION}

The traditional concept of superconductivity is strictly associated with the electron-phonon interaction. The conventional theory is based on the Migdal-Eliashberg adiabatic approximation $^{1}$ that, in fact, leads to the prediction of many peculiar features which are a direct evidence of a phonon mediated superconductivity. The adiabatic approximation is valid if the parameter $\omega_{0} / E_{f}$ is small $\left(\omega_{0}\right.$ is the relevant phonon frequency and $E_{f}$ is the Fermi energy). Usually this parameter is regarded as a measure of nonadiabaticity. However, crossover from a conventional adiabatic to an unconventional nonadiabatic regime does not depend only on the value of the $\omega_{0} / E_{f}$ ratio. Paci et al. ${ }^{8}$ show that even in a case of small "adiabatic" ratio one would expect the nonadiabatic coupling in superconductors having high value of the electron-phonon coupling constant $\lambda_{\text {el-ph}}$. Among BCS superconductors the zirconium dodecaboride $\left(\mathrm{ZrB}_{12}\right)$ is probably a candidate for the observation of such type of anomalous coupling. It stems from the rather small value of the Fermi energy $\sim 1 \mathrm{eV}$ (Ref. 3) that, together with the Debye temperature $\sim 20 \mathrm{meV},{ }^{4}$ leads to a ratio $\omega_{0} / E_{f} \sim 0.02$. A strong coupling ratio $2 \Delta / k_{B} T_{c} \simeq 4.8$ was observed by surface sensitive techniques. ${ }^{3,6}$ This suggests that the electron-phonon coupling constant, which has a bulk value $\lambda_{\text {el-ph }} \simeq 0.67,{ }^{3}$ increases at the surface. From the comparison with strong coupled metallic superconductors ${ }^{7}$ one would expect $\lambda_{\text {el-ph }}^{\text {surf. }}$ $\simeq 1.7-1.9$. Moreover, it was pointed out by Cappelluti et al. ${ }^{2}$ that nonadiabatic character can be further enhanced by low charge carrier density, that is the case for $\mathrm{ZrB}_{12} \cdot{ }^{4,5}$

One of the key features of nonadiabatic superconductivity is the observation of unconventional isotope and pressure effects on the magnetic field penetration depth $\lambda$. Note that
PACS number(s): 74.70.Ad, 74.25.Ha, 74.62.Fj, 83.80.Fg

in adiabatic superconductors (or in the superconductors where the nonadiabatic effects are small) the pressure effect $(\mathrm{PE})^{9,10}$ as well as the isotope effect (IE) ${ }^{11}$ on $\lambda$ was found to be almost negligible in comparison with substantial $\mathrm{PE}^{12}$ and $\mathrm{IE}^{13}$ on $\lambda$ observed in highly nonadiabatic high- $T_{c}$ cuprates. In this paper we report on PE on $T_{c}$ and $\lambda$ studies in $\mathrm{ZrB}_{12}$ superconductor. The magnetic penetration depth measured in the Meissner state is largely determined by the surface characteristics. The absolute value of $\lambda$ at zero temperature and zero pressure was found to be $\lambda(0)=140(30) \mathrm{nm}$. The transition temperature $T_{c}$ and the electron-phonon coupling constant decrease with pressure with the pressure effect coefficients $d T_{c} / d p=-0.0225(3) \mathrm{K} / \mathrm{kbar}$ and $d \ln \lambda_{\text {el-ph }} / d p$ $\simeq-0.2 \% / \mathrm{kbar}$, respectively. In contrast to $T_{c}, \lambda^{-2}(0)$ was found to increase with $d \lambda^{-2}(0) / d p=0.29(11) \mu \mathrm{m}^{-2} / \mathrm{kbar}$. Only a small part of this effect can be explained by a pressure induced renormalization of the electron-phonon interaction and the band structure effects. The major part can be probably explained by considering the possibility of a nonadiabatic coupling of the charge carriers to the crystal lattice appearing in $\mathrm{ZrB}_{12}$ close to the surface.

\section{EXPERIMENTAL DETAILS}

Details on the sample preparation for $\mathrm{ZrB}_{12}$ can be found elsewhere. ${ }^{14}$ The single crystal has been grounded in mortar and then etched in nitric acid in order to remove the inclusion of impurity $\mathrm{ZrB}_{2}$ fraction. ${ }^{15}$ After that, powder was sieved via the $10 \mu \mathrm{m}$ sieve in order to obtain small grains needed for determination of $\lambda$ from magnetization measurements. The grain size distribution was determined by analyzing scanning electron microscope (SEM) photographs. The hydrostatic pressure was generated in a copper-beryllium 


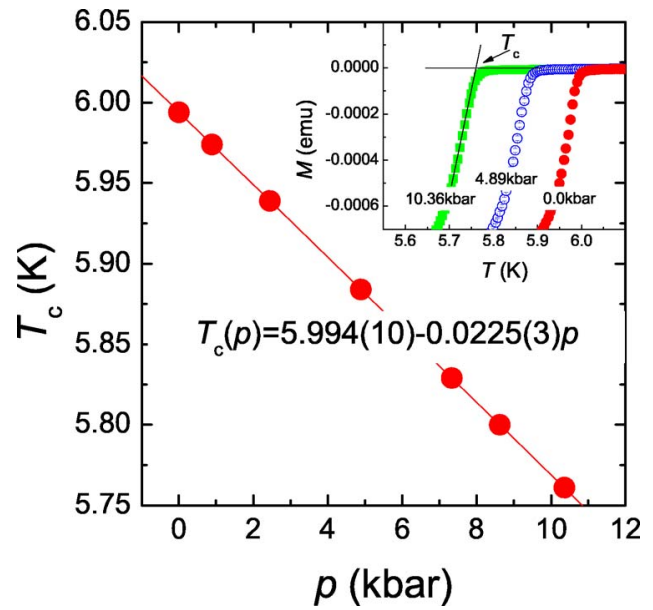

FIG. 1. (Color online) Pressure dependence of the transition temperature $T_{c}$ for $\mathrm{ZrB}_{12}$. The errors are smaller than the size of the symbols. The inset shows $M(T)$ curves in the vicinity of $T_{c}$ for (from the left to the right) $10.36,4.89$, and $0.0 \mathrm{kbar}$.

piston cylinder clamp especially designed for magnetization measurements under pressure. ${ }^{16}$ The sample was mixed with Fluorient FC77 (pressure transmitting medium) with a sample-to-liquid volume ratio of approximately $1 / 6$. The pressure dependence of $T_{c}$ was taken from a separate set of magnetization experiments where a small piece of indium $\left[T_{c}(p=0)=3.4 \mathrm{~K}\right]$ with known $T_{c}(p)$ dependence was added to the sample and both $T_{c}$ 's of indium and $\mathrm{ZrB}_{12}$ were recorded. The field-cooled (FC) magnetization measurements were performed with a SQUID magnetometer in a field of $0.5 \mathrm{mT}$ at temperatures between $1.75 \mathrm{~K}$ and $10 \mathrm{~K}$. The absence of weak links between grains was confirmed by the linear magnetic field dependence of the FC magnetization, measured at $0.25 \mathrm{mT}, 0.5 \mathrm{mT}$, and $1.0 \mathrm{mT}$ for the highest and the lowest pressures at $T=1.75 \mathrm{~K}$.

\section{EXPERIMENTAL RESULTS AND DISCUSSION}

Figure 1 shows the pressure dependence of the transition temperature $T_{c}$ of $\mathrm{ZrB}_{12}$ obtained from magnetization measurements. $T_{c}$ was taken from the linearly extrapolated $M(T)$ curves in the vicinity of $T_{c}$ with $M=0$ line (see inset in Fig. 1). The linear fit yields $d T_{c} / d p$ $=-0.0225(3) \mathrm{K} / \mathrm{kbar}$. Note that this value is in good agreement with $d T_{c} / d p \simeq-0.024 \mathrm{~K} / \mathrm{kbar}$ obtained indirectly by Lortz et al. ${ }^{4}$ from thermal expansion measurements.

The logarithmic volume derivative of $T_{c}$ in the case of a strong coupled BCS superconductor can be described by the following equation: ${ }^{17}$

$$
\frac{d \ln T_{c}}{d \ln V}=-B \frac{d \ln T_{c}}{d p}=(2 A-1) \gamma+A \frac{d \ln \eta}{d \ln V},
$$

where $\quad A=1.04 \lambda_{\text {el-ph }}\left(1+0.38 \mu^{*}\right)\left[\lambda_{\text {el-ph }}-\mu^{*}\left(1+0.62 \lambda_{\text {el-ph }}\right)\right]^{-2}$ is a function of the electron-phonon coupling constant $\lambda_{\text {el-ph }}$ and the Coulomb pseudopotential $\mu^{*},{ }^{17} B$ denotes the bulk modulus, $\gamma=-d \ln \langle\omega\rangle / d \ln V$ is the Grüneisen parameter, $\langle\omega\rangle$ is an average phonon frequency, $\eta \equiv N\left(E_{f}\right)\left\langle I^{2}\right\rangle$ is the Hopfeld parameter. ${ }^{19} N\left(E_{f}\right)$ is the density of states at the Fermi level, and $\left\langle I^{2}\right\rangle$ is the average squared electronic matrix element. Let us now apply Eq. (1) to an analysis of $d T_{c} / d p$ data for $\mathrm{ZrB}_{12}$ superconductor. One should note, however, that not all the quantities needed for such analysis have been measured for $\mathrm{ZrB}_{12}$. Thus we must account for "typical" values or values measured in similar compounds. The Hopfeld parameter $\eta$ generally increases under pressure with $d \ln \eta / d \ln V \approx-1$ for $s$-, and $p$-metal superconductors ${ }^{18}$ and -3 to -4 for transition-metal ( $d$-electron) superconductors. ${ }^{19}$ Since the band structure of zirconium dodecaboride near $E_{f}$ (Ref. 20) as well as the pressure effect on $T_{c}$ look like those of a simple metal, for the Hopfeld parameter we choose $d \ln \eta / d \ln V=-1$. In all conventional superconductors with low critical temperatures the values of $\mu^{*}$ range from 0.1 to 0.15 with most concentrated around 0.1 (see, e.g., Ref. 7). Assuming now $\mu^{*}=0.1$, and taking $\lambda_{\text {el-ph }} \simeq 0.67$ (Ref. 3) and $B=2490 \mathrm{kbar}$ in analogy with $\mathrm{UB}_{12},{ }^{21}$ for the Grüniesen parameter we get the value $\gamma \simeq 2.83$. This value is in reasonable agreement with $\gamma \simeq 3.3$ obtained at a temperature slightly above $T_{c}$ by Lortz et al. ${ }^{4}$ based on thermal expansion measurements. Such an agreement also indicates that the chosen values for the volume derivative of the Hopfeld parameter and the chemical potential are reliable.

$\mathrm{PE}$ on the electron-phonon coupling constant $\lambda_{\text {el-ph }}$ can be determined by using the well-known McMillan equation, ${ }^{22}$

$$
\lambda_{\mathrm{el}-\mathrm{ph}} \propto \frac{N\left(E_{f}\right)\left\langle I^{2}\right\rangle}{\left\langle\omega^{2}\right\rangle},
$$

which leads to

$$
\frac{d \ln \lambda_{\mathrm{el}-\mathrm{ph}}}{d p}=-\frac{1}{B} \frac{d \ln \eta}{d \ln V}-\frac{2 \gamma}{B} .
$$

Substitution of $\gamma=2.83$ and $d \ln \eta / d \ln V=-1 \quad$ gives $d \ln \lambda_{\text {el-ph }} / d p=-0.19 \% /$ kbar. A slightly larger value $d \ln \lambda_{\mathrm{el}-\mathrm{ph}} / d p=-0.22 \% / \mathrm{kbar}$ is obtained with $\gamma=3.3$ from Ref. 4.

As a next step we studied the pressure effect on the magnetic field penetration depth $\lambda$. The temperature dependence of $\lambda$ was calculated from the measured FC magnetization by using the Shoenberg formula, ${ }^{23}$ modified for the known grain size distribution $N(R),{ }^{24}$

$$
\chi=-\frac{3}{2} \int_{0}^{\infty}\left(1-\frac{3 \lambda}{R} \operatorname{coth} \frac{R}{\lambda}+\frac{3 \lambda^{2}}{R^{2}}\right) g(R) d R / \int_{0}^{\infty} g(R) d R,
$$

where $\chi=M / H V$ is the volume susceptibility, $V$ is the volume of the sample, $R$ is the grain radius, and $g(R)$ is the analytical function describing the $N(R) R^{3}$ dependence (see inset in Fig. 2). The resulting temperature dependence $\lambda^{-2}(T)$ at ambient pressure is shown in Fig. 2. The reconstructed data were fitted with the empirical power-law $\lambda^{-2}(T) / \lambda^{-2}(0)=1-\left(T / T_{c}\right)^{n}{ }^{25}$ The fit yields $\lambda^{-2}(0)$ $=48.4(2) \mu \mathrm{m}^{-2}, T_{c}=6.078(5) \mathrm{K}$, and $n=3.65(4)$. Note that the value of the power exponent $n$ is close to " 4 ", which corresponds to a strong-coupled BCS superconductor. ${ }^{26}$ 


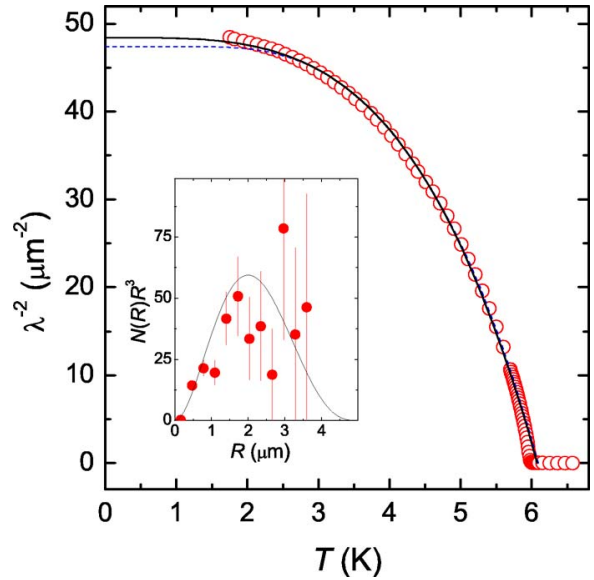

FIG. 2. (Color online) The temperature dependence of $\lambda^{-2}$ calculated from the measured $\chi(T)$ by using Eq. (4). Lines represent fits with the BCS model (dashed line), and with a power law (solid line). See text for an explanation. The inset shows the volume fraction distribution $N(R) R^{3}$ of the $\mathrm{ZrB}_{12}$ powder determined from SEM photographs. The errors are statistical. The solid line represents the analytical $g(R)$ function used in Eq. (4).

In order to obtain the value of the superconducting gap $\Delta$, the data have also been analyzed by means of the BCS model. For clean superconductor the temperature dependence of $\lambda^{-2}$ can be described in the following way: ${ }^{26}$

$$
\frac{\lambda^{-2}(T)}{\lambda^{-2}(0)}=1+2 \int_{\Delta(T)}^{\infty} \frac{\partial F}{\partial E} \frac{E}{\sqrt{E^{2}-\Delta(T)^{2}}} d E
$$

where $F=\left[1+\exp \left(E / k_{B} T\right)\right]^{-1}$ is the Fermi function, $\Delta(T)$ $=\Delta_{0} \cdot \widetilde{\Delta}\left(T / T_{c}\right)$ represents the temperature dependence of the energy gap, and $\Delta_{0}$ is the zero temperature value of the superconducting gap. $\widetilde{\Delta}\left(T / T_{c}\right)$ is the normalized gap taken from Ref. 27. The best fit to the data using Eq. (5) gives $T_{c}=6.09(2) \mathrm{K}, \lambda^{-2}(0)=47.4(2) \mathrm{nm}$, and $\Delta_{0}=1.251(9) \mathrm{meV}$. The ratio $2 \Delta_{0} / k_{B} T_{c}=4.77(4)$ is found, suggesting that $\mathrm{ZrB}_{12}$ is a strong coupled BCS superconductor. Note that a rather close value $2 \Delta_{0} / k_{B} T_{c} \simeq 4.8$ has been obtained in pointcontact spectroscopy ${ }^{3}$ and tunnelling ${ }^{6}$ experiments. On the other hand, a smaller value $\simeq 3.7$ has been reported by Lortz et $a l .{ }^{4}$ using the heat-capacitance technique, thus suggesting a weak coupling strength. This difference has been already pointed out by Tsindlekht et al. ${ }^{6}$ It was explained by enhanced surface characteristics of the $\mathrm{ZrB}_{12}$ leading to rather different superconducting properties of bulk $4,6,28$ and surface. ${ }^{3,6,29,30}$ Our measurements were performed in the Meissner state, with the field penetrating on a distance $\lambda$ from the surface and, therefore, give a value of the superconducting gap consistent with those reported in the surface sensitive experiments. ${ }^{3,6}$ To estimate the uncertainty in the absolute value of $\lambda(0)$ we used a procedure similar to that one described in Refs. 9 and 12. The temperature dependence of $\lambda(T)$ was calculated for $N(R)+\sqrt{N(R)}$ and $N(R)-\sqrt{N(R)}$ distributions. The fit of the resulting $\lambda(T)$ curves with the power law as well as with the BCS model gives $\lambda(0)$ in the range from 110 to $170 \mathrm{~nm}$. Note that the values of the power ex-
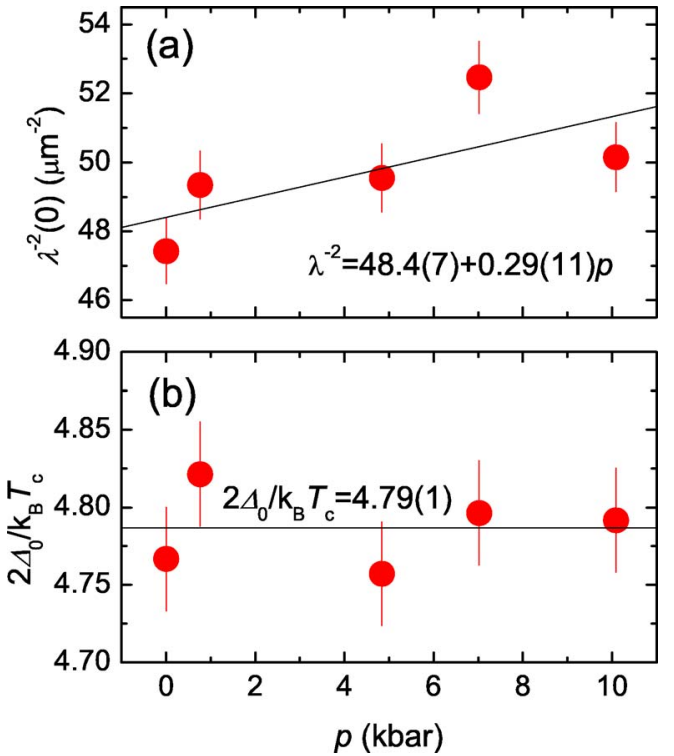

FIG. 3. (Color online) Pressure dependences of $\lambda^{-2}(0)$ (a) and $2 \Delta_{0} / k_{B} T_{c}(\mathrm{~b})$. The solid lines are fits with parameters shown in the figures.

ponent $n$ (zero temperature superconducting gap $\Delta_{0}$ ) were found to be well within error bars for each series of $\lambda(T)$ curves.

Figure 3(a) shows the pressure dependence of $\lambda^{-2}(0)$ obtained by fitting the reconstructed $\lambda(T)$ data at different pressures with the BCS model [Eq. (5)]. In these experiments we studied relative effects measured on the same sample in the same pressure cell. The main systematic error of these measurements comes from misalignments of the experimental setup occurring when the cell is removed from the SQUID magnetometer, to change the pressure, and put back again. This procedure was checked with a set of measurements at constant pressure. The systematic scattering of the magnetization data is about $0.3 \%$, giving a relative error in $\lambda^{-2}(T)$ of about $3 \%$. The reducing of the grain size with pressure was taken into account in $\lambda(T)$ calculation [Eq. (4)], by using the bulk modulus reported above. The linear fit yields $\lambda^{-2}(0)$ $=48.4(7)+0.29(11) p$, implying that $\lambda^{-2}$ increases under pressure with $d \ln \lambda^{-2}(0) / d p=0.60(23) \% /$ kbar [see Fig. 3(a)].

To analyze the observed effect we used a procedure similar to the one described by Di Castro et al. ${ }^{10}$ There it was suggested that $\lambda^{-2}$ increases under pressure because of two reasons, (i) band structure effects and (ii) renormalization of the electron-phonon coupling. ${ }^{10}$ Under the assumption of ellipsoidal or cylindrical Fermi surface the first one can be obtained as ${ }^{10}$

$$
\frac{d \ln \lambda^{-2}(0)}{d p}=\frac{1}{3 B}-\frac{d \ln N\left(E_{f}\right)}{d p} \simeq \frac{1}{3 B}-\frac{1}{B} \frac{d \ln \eta}{d \ln V} .
$$

Here we used the fact that the pressure dependence of the electronic matrix element $\left\langle I^{2}\right\rangle$ entering the Hopfeld parameter $\eta$ can usually be neglected. ${ }^{31}$ Hence, by setting $d \ln \eta / d \ln V \simeq-1$, and $B=2490$ kbar (see above) we obtain $d \ln \lambda^{-2}(0) / d p=0.05 \% /$ kbar. 
The electron-phonon renormalized penetration depth reduces to $\lambda^{*-2}(0)=\lambda^{-2}(0) /\left(1+\lambda_{\text {el-ph }}\right),{ }^{7}$ where $\lambda(0)$ is the bare quantity we have considered before. We have then

$$
\frac{d \ln \lambda^{*-2}(0)}{d p}=-\frac{\lambda_{\mathrm{el}-\mathrm{ph}}}{1+\lambda_{\mathrm{el}-\mathrm{ph}}} \frac{d \ln \lambda_{\mathrm{el}-\mathrm{ph}}}{d p} .
$$

By substituting $\lambda_{\text {el-ph }} \simeq 0.67$ (Ref. 3) and $d \ln \lambda_{\text {el-ph }} / d p$ $\simeq-0.2 \% / \mathrm{kbar}$ obtained above we get $d \ln \lambda^{*-2}(0) / d p$ $\simeq 0.08 \% / \mathrm{kbar}^{32}$ Thus the total pressure shift of $\lambda^{-2}(0)$ expected assuming conventional (adiabatic) coupling of the charge carriers to the lattice in $\mathrm{ZrB}_{12}$ is of the order of $0.13 \% / \mathrm{kbar}$. This value is more than three times smaller than the experimentally observed one $0.60(23) \% / \mathrm{kbar}$. This implies that in addition to band structure effects and renormalization of the electron-phonon coupling there are other effects responsible for the increasing of $\lambda^{-2}(0)$ under pressure. Bearing in mind that $\lambda$ measurements have been performed in a Meissner state, the observed dependence of $\lambda$ on $p$ can be explained suggesting that in $\mathrm{ZrB}_{12}$ close to the surface the coupling of the charge carriers to the lattice has a nonadiabatic character. Note that similar effects have been observed in $\mathrm{YBa}_{2} \mathrm{Cu}_{4} \mathrm{O}_{8}$ that appears to be a highly nonadiabtic superconductor. ${ }^{12}$

The results on the zero temperature superconducting gap $\Delta_{0}$ are summarized in Fig. $3(\mathrm{~b})$, where the ratio $2 \Delta_{0} / k_{B} T_{c}$ is plotted as a function of the pressure $p . \Delta_{0}$ and $T_{c}$ were obtained from the fit of $\lambda^{-2}(T, p)$ data by using Eq. (5). The solid line represents a fit by the relation $2 \Delta_{0} / k_{B} T_{c}=$ const to the data. Bearing in mind that $T_{c}$ scales linearly with pressure (see Fig. 1) the constant ratio can be understood in the frame of the BCS theory, which predicts $2 \Delta_{0} / k_{B} T_{c}=3.52$. In the present study this ratio was found to be pressure independent within experimental errors, with mean value 4.79(1).

\section{CONCLUSIONS}

In conclusion, we performed magnetization measurements in $\mathrm{ZrB}_{12}$ under hydrostatic pressure. A negative pressure effect on $T_{c}$ with $d T_{c} / d p=-0.0225(3) \mathrm{K} / \mathrm{kbar}$ is observed. The electron-phonon coupling constant $\lambda_{\text {el-ph }}$ decreases with pressure with $d \ln \lambda_{\text {el-ph }} / d p \simeq-0.20 \% / \mathrm{kbar}$. The magnetic field penetration depth $\lambda$ measured in the Meissner state is largely determined by the surface characteristics. ${ }^{33} \lambda$ was found to increase with pressure, with the pressure effect coefficient $d \ln \lambda^{-2}(0) / d p=0.60(23) \% / \mathrm{kbar}$. This coefficient is much larger than that one estimated theoretically within the adiabatic approximation. This can be explained suggesting that in $\mathrm{ZrB}_{12}$, close to the surface, the coupling of the charge carriers to the lattice has a nonadiabatic character. The ratio $2 \Delta_{0} / k_{B} T_{c}=4.79(1)$ is found to be pressure independent and close to the strong coupling BCS value 4.8(1) reported in Refs. 3 and 6 . The value of $\lambda$ extrapolated to zero temperature and at $p=0$ was estimated to be $140(30) \mathrm{nm}$.

\section{ACKNOWLEDGMENTS}

The authors are grateful to S. Strässle for help during the preparation of this paper. This work was supported by the Swiss National Science Foundation and by the NCCR program Materials with Novel Electronic Properties (MaNEP) sponsored by the Swiss National Science Foundation.
${ }^{1}$ A. B. Migdal, Sov. Phys. JETP 7, 996 (1958); G. M. Eliashberg, ibid. 11, 696 (1960).

${ }^{2}$ E. Cappelluti and L. Pietronero, Phys. Status Solidi B 242, 133 (2005).

${ }^{3}$ D. Daghero, R. S. Gonnelli, G. A. Ummarino, A. Calzoilari, V. Dellarocca, V. A. Stepanov, V. B. Filippov, and Y. B. Paderno, Supercond. Sci. Technol. 17, S250 (2004).

${ }^{4}$ R. Lortz, Y. Wang, S. Abe, C. Meingast, Yu. B. Paderno, V. Filippov, and A. Junod, Phys. Rev. B 72, 024547 (2005).

${ }^{5}$ B. T. Matthias, T. H. Geballe, K. Andres, E. Corenzwit, G. W. Hull, and J. P. Maita, Science 159, 530 (1968).

${ }^{6}$ M. I. Tsindlekht, G. I. Leviev, I. Asulin, A. Sharoni, O. Millo, I. Felner, Y. B. Paderno, V. B. Filippov, and M. A. Belogolovskii, Phys. Rev. B 69, 212508 (2004).

${ }^{7}$ J. P. Carbotte, Rev. Mod. Phys. 62, 1027 (1990).

${ }^{8}$ P. Paci, M. Capone, E. Cappelluti, S. Ciuchi, C. Grimaldi, and L. Pietronero, Phys. Rev. Lett. 94, 036406 (2005).

${ }^{9}$ R. Khasanov, D. G. Eshchenko, J. Karpinski, S. M. Kazakov, N. D. Zhigadlo, R. Brütsch, D. Gavillet, D. Di Castro, A. Shengelaya, F. La Mattina, A. Maisuradze, C. Baines, and H. Keller, Phys. Rev. Lett. 93, 157004 (2004).

${ }^{10}$ D. Di Castro, R. Khasanov, C. Grimaldi, J. Karpinski, S. M. Kazakov, R. Brütsch, and H. Keller, Phys. Rev. B 72, 094504 (2005).
${ }^{11}$ D. Di Castro, M. Angst, D. G. Eshchenko, R. Khasanov, J. Roos, I. M. Savić, A. Shengelaya, S. L. Budko, P. C. Canfield, K. Conder, J. Karpinski, S. M. Kazakov, R. A. Ribeiro, and H. Keller, Phys. Rev. B 70, 014519 (2004).

${ }^{12}$ R. Khasanov, J. Karpinski, and H. Keller, J. Phys.: Condens. Matter 17, 2453 (2005).

${ }^{13}$ G. M. Zhao, M. B. Hunt, H. Keller, and K. A. Müller, Nature (London) 385, 236 (1997); J. Hofer, K. Conder, T. Sasagawa, G.-M. Zhao, M. Willemin, H. Keller, and K. Kishio, Phys. Rev. Lett. 84, 4192 (2000); R. Khasanov, D. G. Eshchenko, H. Luetkens, E. Morenzoni, T. Prokscha, A. Suter, N. Garifianov, M. Mali, J. Roos, K. Conder, and H. Keller, ibid. 92, 057602 (2004); R. Khasanov, A. Shengelaya, E. Morenzoni, K. Conder, I. M. Savić, and H. Keller, J. Phys.: Condens. Matter 16, S4439 (2004).

${ }^{14}$ Y. B. Paderno, A. B. Liashchenko, V. B. Filippov, and A. V. Dukhnenko, Proceedings of the International Conference on Science for Materials in the Frontier of the Centuries: Advantages and Challenges (IPMS NASU, Kiev, 2002), p. 347).

${ }^{15}$ V. A. Gasparov, N. S. Sidorov, and I. I. Zver'kova, cond-mat/ 0508151 (unpublished).

${ }^{16}$ T. Straessle, Ph.D. thesis, ETH, Zurich, 2001.

${ }^{17}$ T. Tomita, J. J. Hamlin, J. S. Schilling, D. G. Hinks, and J. D. Jorgensen, Phys. Rev. B 64, 092505 (2001). 
${ }^{18}$ J. S. Schilling and S. Klotz, Physical Properties of High Temperature Superconductors, Vol. III (World Scientific, Singapore, 1992), p. 59.

${ }^{19}$ J. J. Hopfeld, Physica (Utrecht) 55, 41 (1971).

${ }^{20}$ I. R. Shein and A. L. Ivanovskii, Phys. Solid State 45, 1429 (2003).

${ }^{21}$ J.-P. Dancausse, E. Gering, S. Heathman, U. Benedict, L. Gerward, S. S. Olsen, and F. Hulliger, J. Alloys Compd. 189, 205 (1992).

${ }^{22}$ W. L. McMillan, Phys. Rev. 167, 331 (1968).

${ }^{23}$ D. Shoenberg, Proc. R. Soc. London, Ser. A 175, 49 (1940).

${ }^{24}$ A. Porch, J. R. Cooper, D. N. Zheng, J. R. Waldram, A. M. Campbell, and P. A. Freeman, Physica C 214, 350 (1993).

${ }^{25}$ P. Zimmermann, H. Keller, S. L. Lee, I. M. Savić, M. Warden, D. Zech, R. Cubitt, E. M. Forgan, E. Kaldis, J. Karpinski, and C. Krúger, Phys. Rev. B 52, 541 (1995).

${ }^{26}$ M. Tinkham, Introduction to Superconductivity (Krieger, Malabar, FL, 1975)

${ }^{27}$ B. Mühlschlegel, Z. Phys. 155, 313 (1959).

${ }^{28}$ Y. Wang, R. Lortz, Yu. Paderno, V. Filippov, S. Abe, U. Tutsch, and A. Junod, Phys. Rev. B 72, 024548 (2005).
${ }^{29}$ G. I. Leviev, V. M. Genkin, M. I. Tsindlekht, I. Felner, Y. B. Paderno, and V. B. Filippov, Phys. Rev. B 71, 064506 (2005).

${ }^{30}$ V. A. Gasparov, N. S. Sidorov, I. I. Zver'kova, S. S. Khassanov, and M. P. Kulakov, JETP 101, 98 (2005).

${ }^{31}$ B. Lorenz and C. W. Chu, in Frontiers in Superconducting Materials, edited by A. V. Narlikar (Springer, Berlin, Heidelberg, 2005), p. 459.

${ }^{32}$ Assuming for $\lambda_{\text {el-ph }}$ the surface enhanced value 1.9 (see introduction) we obtain a slightly larger value $d \ln \lambda_{\mathrm{el}-\mathrm{ph}} / d p$ $\simeq 0.13 \% / \mathrm{kbar}$.

${ }^{33}$ Generally properties within $140 \mathrm{~nm}$ from the surface are assumed to be bulk. However, difference between "bulk" and "surface" notations depends strongly on the physical phenomenon studied. The length where superconducting properties are changed is the coherence length. It follows from direct magnetization measurements by Tsindlekht et al. (Ref. 6) and Wang et al. (Ref. 28) that in the case of $\mathrm{ZrB}_{12}$ the coherence length is of the order of the magnetic penetration depth. That is why we consider the properties within the depth of $140 \mathrm{~nm}$ as superconducting surface ones. 